\title{
Linkages between hydrologic cycling and hydro-biogeochemical processes in salt marsh systems
}

\author{
MARGARET ZIMMER ${ }^{1}$, MAYA MONTALVO ${ }^{1}$, EMILIO \\ GRANDE $^{1}$, CORIANNE TATARIW ${ }^{2}$, ERIN SEYBOLD ${ }^{3}$, \\ ANNA BRASWELL ${ }^{4}$, ALICE KLEINHUIZEN ${ }^{1}$, ATE \\ VISSER $^{5}$ AND BHAVNA ARORA ${ }^{6}$ \\ ${ }^{1}$ University of California, Santa Cruz \\ ${ }^{2}$ University of Alabama \\ ${ }^{3}$ University of Kansas \\ ${ }^{4}$ University of Florida \\ ${ }^{5}$ Lawrence Livermore National Laboratory \\ ${ }^{6}$ Lawrence Berkeley National Laboratory \\ Presenting Author: margaret.zimmer@ucsc.edu
}

Salt marshes exist at the tidal interface between watersheds and the ocean and are hot spots of biogeochemical activity within coastal watersheds. Yet, we have limited understanding about how short-term (e.g. daily tidal cycles) and long-term (e.g. seasonal precipitation and climate patterns) hydrologic forcings may interact within marshes. These interactions will affect the temporal dynamics, or hot moments, of nutrient processing within these systems as well as the physical zonation of biogeochemical processing in the subsurface, or hot spots. To address this knowledge gap, we instrumented a $25 \mathrm{~m}$ transect along a representative salt marsh system at the Elkhorn Slough National Estuarine Research Reserve in California, USA. We installed variable-depth redox probes, nested wells and piezometers, and a field-deployable spectrophotometer with a multi-source pump at lower, mid-, and upper marsh positions to allow for high frequency characterization of subsurface hydrologic cycling and dissolved inorganic nitrogen (DIN) species concentrations. We also conducted seasonal sediment incubation experiments to quantify nitrogen processing rates. We found that DIN concentrations fluctuated daily due to frequent tidal flushing that introduced oxygen and ammonium-rich surface water into sediments under reduced conditions, with the largest daily change in concentrations observed in lower marsh positions. Sediment core incubations consistently showed net $\mathrm{N}_{2}$ flux out of the marsh, indicating removal by denitrification, but the impacts of seasonal and event-driven freshwater contributions affected elevations differently, with the largest changes seen in upper marsh positions. Together, our findings suggest that intra-annual changes in source water contributions across the salt marsh result in functional zonation, where lower marsh position functions are regulated by tidal flushing and upper marsh position functions are regulated by freshwater contributions. 